\title{
A double-blind controlled trial of etretinate (Tigason) and ibuprofen in psoriatic arthritis
}

\author{
R HOPKINS, H A BIRD, H JONES, J HILL, K E SURRALL, C ASTBURY, \\ ${ }^{*}$ A MILLER, AND V WRIGHT
}

From the Clinical Pharmacology Unit, Royal Bath Hospital, Harrogate, and the General Infirmary at Leeds, and ${ }^{*}$ Roche Products Limited, Welwyn Garden City, Hertfordshire

SUMMARY Etretinate (Tigason) and ibuprofen have been compared in a double-blind controlled trial in psoriatic arthritis to see if we could confirm a specific action for this vitamin A derivative suggested from earlier uncontrolled studies. Eleven out of 20 patients completed 24 weeks of therapy with etretinate (up to $0.5 \mathrm{mg} / \mathrm{kg} /$ day) whereas only $1 / 20$ patients completed 24 weeks of therapy with ibuprofen alone. Etretinate improved skin lesions, and this may have encouraged patients to persist with it. Improvement of statistical significance was seen for articular index in both groups. In addition significant improvement in ESR, haemoglobin, C-reactive protein, and histidine occurred in the etretinate group. The main side effects of etretinate (which may preclude its use at a higher dose in this condition) included cracked and dried lips and sore mouth.

Etretinate (Tigason) is a vitamin A derivative. The parent vitamin is essential for growth, maintenance of visual function, and the regulation of proliferation and differentiation of epithelial tissues. Because of the effect on the epithelium, vitamin A derivatives have been used in dermatology for the treatment of acne, psoriasis, and other skin conditions. ${ }^{1}$ About 1500 retinoids have been synthesised and biologically tested in the last 15 years. ${ }^{2}$ Etretinate (an aromatic retinoid) has a therapeutic index 10 times more favourable than the parent all-transretinoic acid in the treatment of skin conditions, and excellent improvements have been achieved with this compound in generalised and refractory pustular forms of psoriasis. ${ }^{3}$

Controlled trials of etretinate by dermatologists that demonstrate its efficacy clinically, histologically, and immunologically in psoriatic skin lesions make no mention of a possible action on the joints. ${ }^{4}$ Subsequently three open studies by rheumatologists claimed efficacy in psoriatic arthropathy. Stollenwerk et al. treated 24 patients for 6-18 months. They claimed clinical improvement in joints, serial im-

Accepted for publication 13 September 1984.

Correspondence to Dr H A Bird, Clinical Pharmacology Unit (Rheumatism Research), Royal Bath Hospital, Cornwall Road, Harrogate HG1 2PS. provement in ${ }^{99 m}$ technetium scans of the hands and feet, and 'no progression of destructive change' on $x$-rays. ${ }^{5}$ Rosenthal et al. treated four patients for nine months, noting clinical improvement and a fall in ESR. ${ }^{6}$ Bitter et al. treated 32 patients for 6-18 months and noted clinical and immunological improvement as well as a fall in ESR. ${ }^{7}$

In view of the immunosuppressive, immunostimulant, collagenase inhibition, and free oxygen radical inhibition properties of etretinate in animal models it was thought appropriate to conduct a controlled trial of etretinate against ibuprofen (an accepted non-steroidal anti-inflammatory agent) in psoriatic arthritis. Plasma viscosity, C-reactive protein, serum sulphydryl and histidine were added to conventional clinical assessments to confirm the reported effect of the drug on acute phase reactants.

\section{Patients and methods}

The patients were between 18 and 70 years, with joint pain involving three or more joints (proximal interphalangeal (PIP) joints and metacarpophalangeal (MCP) joints on one hand being considered as a single joint) and clinical evidence of inflammatory polyarthritis affecting at least three joints including involvement of the hand, wrist, or foot. Patients had undoubted skin lesions of psoriasis and 
were seronegative for rheumatoid factor (sheep cell agglutination test (SCAT) 1/16 or less).

Exclusion criteria included known sensitivity or allergy to any trial drug, peptic ulcer or other systemic disorders, significant laboratory abnormality, hyperlipidaemia (clinical or laboratory); and patients who had received a 'second-line agent' for their arthritis (gold, D-penicillamine, cytotoxic drugs, sulphasalazine) within the three-month period prior to the trial. A stable dose of corticosteroids was not an exclusion criterion.

Informed consent was obtained from each subject, and local ethical committee approval was obtained. In view of the teratogenic potential of vitamin A derivatives women of childbearing age who wished to participate in the study signed a form agreeing to remain on the contraceptive pill while taking the drug and for two years after discontinuing it.

The study was of double-blind, double dummy parallel group design. The duration was 24 weeks. Patients were allocated at random to either ibuprofen at a constant dose of $400 \mathrm{mg}$ four times a day throughout the study or to etretinate. The starting dose for all patients on etretinate was $0.5 \mathrm{mg} / \mathrm{kg} /$ day rounded up or down to the nearest $10 \mathrm{mg}$, taken as a single morning dose. After four weeks patients experiencing side effects were allowed to reduce to $0.25 \mathrm{mg} / \mathrm{kg} /$ day (to the nearest $10 \mathrm{mg}$ ) or to continue, or to increase to a higher dose if improvement had not occurred. An appropriate double dummy technique was followed throughout the study to maintain blindness. So far as possible all other drug therapy and physiotherapy were left unaltered throughout the 24 weeks of the study.

Patients attended a special clinic at $0,2,4,8,12$, 16,20 , and 24 weeks. On each occasion the following clinical assessments were performed: pain (10 $\mathrm{cm}$ visual analogue scale); early morning stiffness (measured in minutes); global index of improvement (5 point scale); Ritchie articular index ${ }^{8}$; grip strength (mean from both hands measured in mmHg with a sphygmomanometer cuff); proximal interphalangeal joint size (arthrocircometer).

At each visit blood was taken for the following laboratory tests: full blood count; biochemical profile, including renal and hepatic function, serum triglycerides and cholesterol (every 12 weeks); erythrocyte sedimentation rate; plasma viscosity; C-reactive protein; histidine; serum sulphydryl.

Side effects were also sought by the standard question 'Have the tablets upset you in any way?'

Statistical analysis was by Student's $t$ test for the majority of clinical parameters and Wilcoxon or Kruskal-Wallis tests for the biochemical parameters.

\section{Results}

Forty patients entered the study. All conformed to $\stackrel{\vec{F}}{\stackrel{\vec{S}}{+}}$ the entry criteria. Nineteen were male and 21 을 female. The mean age of the group was $49 \cdot 1$ years. 음 The mean ESR for the group on starting the study $\frac{\bar{D}}{\vec{D}}$ was $37 \mathrm{~mm} \mathrm{~h}^{-1}$. Twenty patients were allocated at $\stackrel{\mathbb{Q}}{\Omega}$ random to etretinate, the other twenty to ibuprofen. Both groups were well matched in demographic $\overrightarrow{0}$ characteristics, and there was no significant difference between the two groups for any clinical or $\vec{\omega}$ laboratory parameter of disease activity (Tables 1 and 2).

Eleven out of 20 patients completed 24 weeks of therapy on etretinate. Only $1 / 20$ patients completed $\stackrel{\vec{\omega}}{\vec{c}}$ 24 weeks of therapy on ibuprofen. At eight weeks $12 / 20$ patients remained on ibuprofen, and by $12 \%$ weeks $8 / 20$ patients remained on this drug. The 의 corresponding figures for etretinate were $19 / 20$ and $18 / 20$ respectively.

The main reason for dropout from the ibuprofen group was lack of efficacy. Patients withdrawing $\supset$ from the ibuprofen group for this reason were $\overrightarrow{0}$ offered the option of starting on etretinate, and a or majority elected to do so. The results from this group of patients after changing to etretinate were comparable (both in clinical and biochemical terms) to those obtained from patients initially allocated to etretinate but are not included in this analysis. The $\frac{\circ}{\Phi}$ majority of patients changing from ibuprofen to etretinate on grounds of poor efficacy changed at $\overrightarrow{\vec{O}}$ week $8(7 / 20)$ or at week $12(7 / 20)$. Only one patient discontinued ibuprofen because of side effects (severe nausea).

Two out of 20 patients discontinued etretinate because of lack of efficacy and 7/20 patients discon- $\frac{0}{\sigma}$ tinued because of side effects. The side effects 3 . encountered together with the dose at withdrawal were dyspepsia and lack of efficacy (week 4; 20 을 $\mathrm{mg} /$ day); mouth ulcers, cracked lips, and dyspepsia $\circ$ (week $8,30 \mathrm{mg} /$ day); cracked lips and flare of psoriasis (week $8 ; 40 \mathrm{mg} /$ day); acute exacerbation of skin lesions of psoriasis (week 12;40 mg/day); irritation of skin, sore mouth, dry lips (week 16;20 $\mathrm{mg} /$ day); cracking of finger nails (week 16; $50 \mathrm{~N}$ mg/day); and sore eyes, cracked lips, scaling skin, N hair loss, and alleged impotence (week 20; 50 음 $\mathrm{mg} /$ day).

The majority of patients treated with etretinate had risen to a dose of approximately $0.75 \mathrm{mg} / \mathrm{kg} /$ day by the end of the study. Almost all patients on etretinate noticed an improvement of the psoriatic skin lesions on starting the drug. This may in part have contributed to their perseverance in remaining $\frac{\text { }}{\mathrm{O}}$ on the drug for the full 24 weeks of the trial.

Table 1 shows clinical changes occurring in both 

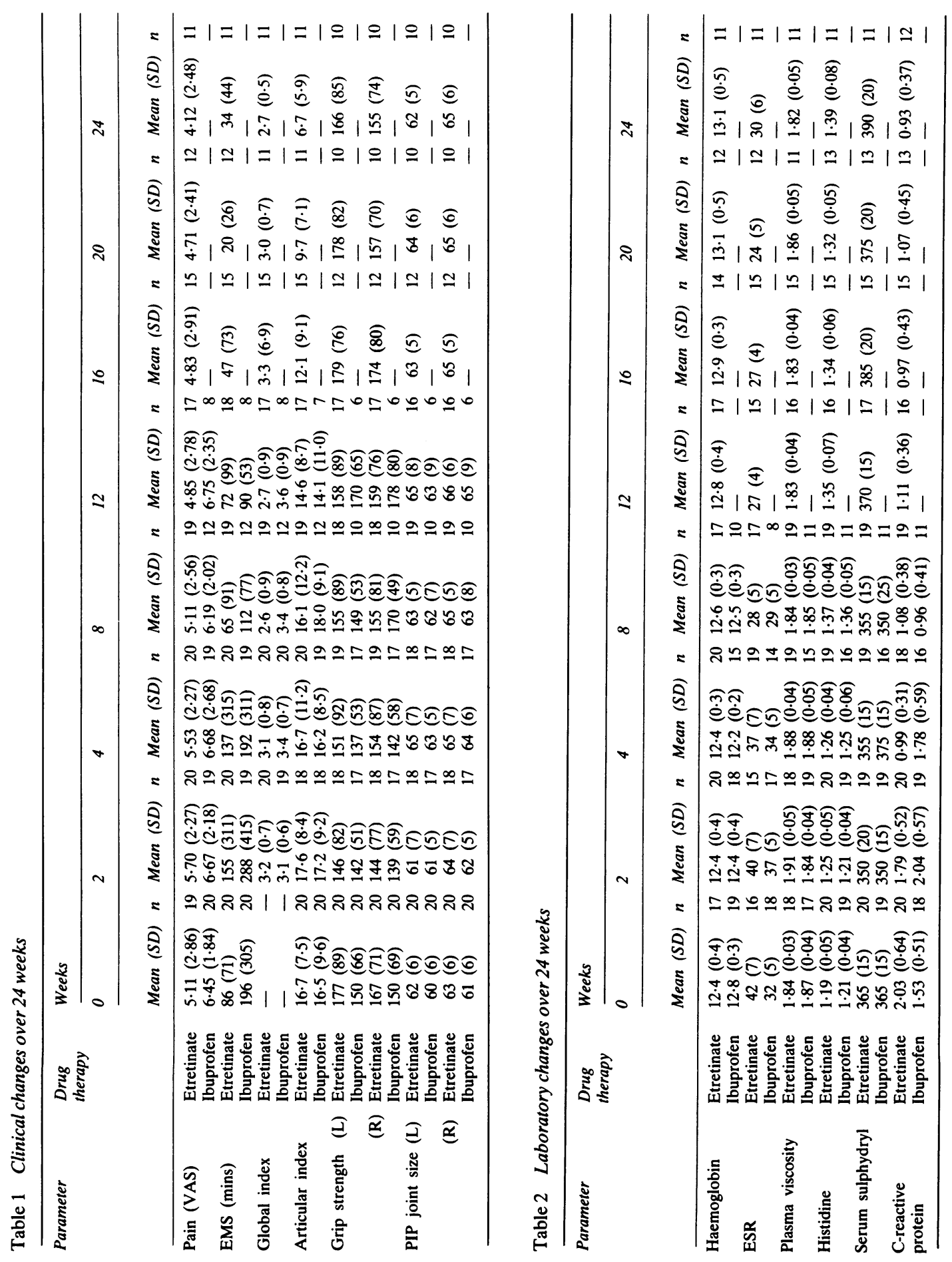
groups through the trial. The table depicts mean patient data at each clinic visit with standard deviation and the number of patients from which these data points are derived. An apparent mismatch at week 0 for early morning stiffness (86 minutes etretinate/196 minutes ibuprofen) did not reach statistical significance. Analysis was discontinued at week 16 for the ibuprofen group in view of diminishing patient numbers. A significant improvement occurred in the ibuprofen-treated group at 12 weeks for articular index $(\mathrm{p}<0 \cdot 01)$, but a trend to improvement in early morning stiffness by eight weeks did not reach significance. Other clinical variables were largely unaltered.

In the etretinate-treated group there was a significant deterioration in early morning stiffness at week $4(p<0.01)$ but a significant improvement at 16 weeks in articular index $(p<0 \cdot 01)$. A gradual improvement in a majority of parameters over the 24-week period did not reach statistical significance. Statistical analysis between patient groups showed no advantage for either treatment at any point, though the larger number of patients able to tolerate etretinate without withdrawal for a full 24 weeks is relevant.

Table 2 shows biochemical changes occurring in both groups throughout the trial. This depicts mean patient data at each clinic visit with standard error of the mean and the number of patients from which this is derived. Slight differences in numbers between Tables 1 and 2 reflect patients who were unable to attend for assessments but from whom blood was collected or losses of laboratory samples. Both groups showed active disease on all laboratory assessments. In the ibuprofen group a significant increase occurred in histidine at week $8(\mathrm{p}<0.05)$, denoting improved disease activity. There was otherwise little overall change during the first eight weeks for which it was possible to make valid statistical comparison before falling numbers precluded further analysis.

In the etretinate-treated group significant improvement occurred in haemoglobin $(\mathrm{p}<0.05$ at 16 and 20 weeks and $p<0.01$ at 24 weeks), ESR $(p<0.05$ at 20 weeks), C-reactive protein ( $p<0.05$ at 16 weeks), and histidine, which reverted upwards towards normality $(p<0.01$ at $8,12,16$, and 20 weeks). Analytical comparison between groups showed no significant advantage of one treatment over the other during the eight weeks of the study that numbers allowed such comparison.

Although serum cholesterol and triglycerides became elevated in a small number of male patients on etretinate, this did not necessitate their withdrawal from the study, and values returned to normal on withdrawal of the drug. Other biochemical parameters were unaltered.

\section{Discussion}

The high dropout rate from the NSAID (ibuprofen) $\frac{\overline{\frac{D}{D}}}{\overparen{D}}$ group was not anticipated when the study was designed and makes interpretation rather difficult. It remains a possibility that the improvement in skin $\vec{\circ}$ lesions seen by patients allocated to etretinate encouraged better compliance than in the ibuprofen $\vec{\sigma}$ group. The clinical changes seen over 24 weeks with etretinate were not striking, and the small numbero of ibuprofen patients available for comparison + towards the end of this period makes it hard to be certain this modest improvement did not arise from $\vec{\infty}$ natural disease remission. Patients inevitably are included in the trial when their disease is at its worst, necessitating referral to clinic.

In spite of this we think etretinate was more efficacious than ibuprofen. It is hard to account for⿳亠口冋 the perseverence of the etretinate group to 24 weeks $\vec{乛}$ unless their joints were ameliorated more than the $\infty$ ibuprofen group. Significant biochemical improve-or ment occurred over the 24 weeks of etretinate treatment, and we think this unlikely to be spontaneous remission alone, since biochemical im-⿳亠二口犬 provement was seen in the ibuprofen 'non- 0 responders' who elected to transfer to etretinate. $\stackrel{\square}{\varrho}$ Thus for those patients whose biochemical para- $\overrightarrow{\vec{A}}$ meters remained unaltered during eight or 12 weeks 3 of ibuprofen therapy, a fall in CRP from a mean of $\bar{\partial}$ 1.56 to 0.67 , and a rise in histidine from a mean of 1.31 to 1.40 was seen during the first 12 weeks of therapy.

Our results provide modest confirmation of $\frac{\dot{2}}{3}$ earlier uncontrolled reports that etretinate is effective in psoriatic arthritis. These earlier results do not $O$ enable one to ascertain whether these patients elected to remain on the drug because of improve-을 ment in the skin. Certainly we have confirmed the $>$ biochemical improvement in disease activity that has previously been reported with this drug ${ }^{6}{ }^{7}$ and think $N$ this is unlikely to be explicable entirely in terms of spontaneous remission. It remains a possibility that 0 we evaluated the drug at too low a dose, though we $\mathrm{N}$ feel the frequency of side effects seen would have? militated against its use at a higher dose in thise condition.

The authors thank Mrs D K Smith for secretarial assistance.

\section{References}

1 Bollag W. Vitamin A and retinoids: from nutrition to pharmacotherapy in dermatology and oncology. Lancet 1983; i: 860-3. 
2 Mayer H, Bollag W, Hanni R, Rvegg R. A new class of compounds with prophylactic and therapeutic activities in oncology and dermatology. Experientia 1978; 34: 1105-19.

3 Peck G L. Retinoids in clinical dermatology. In: Fleischmayer R, ed. Progress in diseases of the skin. New York: Grune and Stratton. 1981: 1: 227-69.

4 Jablonska S, Wolska H, Dabrowski J, Haftek M, Groniowska M, Jarzabek-Chorzelska. Aromatic retinoids in psoriasis: clinical, histological, biochemical, electron microscopical and immunological investigations. In: Orfanos C E, et al., eds. Retinoids, Berlin: Springer, 1981: 165-73.
5 Stollenwerk R, Fischer-Hoinkes H, Komenda K, Schilling F. Clinical observations in oral retinoid therapy for psoriatic arthropathy (Ro10-9359). In: Orfanos C E, et al., eds. Retinoids, Berlin: Springer, 1980: 205-9.

6 Rosenthal M. Retinoid in der Behandling var PsoriasisArthritis. Schweiz Med Wochenschr 1979; 109: 1912-4.

7 Bitter T, Bahous I, Rosenthal M. All-trans retinoic acid (R): a remission aim in the treatment of psoriatic arthritis (PA). Ann Rheum Dis 1981; 40: 209.

8 Ritchie D M. Boyle J A. McInnes J M, et al. Clinical studies with an articular index for the assessment of joint tenderness in patients with rheumatoid arthritis. $Q J$ Med 1968; 37: 393-406. 